\title{
A multicenter clinical study: personalized medication for advanced gastrointestinal carcinomas with the guidance of Patient-Derived Tumor Xenograft (PDTX)
}

\section{Yuan Cheng ( $\square$ 516953980@qq.com)}

Bayi Hospital Affiliated Hospital of Nanjing University of Chinese Medicine https://orcid.org/00000003-2571-4547

\section{Shu-kui Qin}

Bayi Hospital Affiliated to Nanjing University of Chinese Medicine

Jin Li

Shanghai East Hospital

\section{Guang-hai Dai}

Chinese PLA General Hospital

\section{Bai-yong Shen}

Shanghai Ruijin Hospital

Jieer Shen

Zhejiang Cancer Hospital

\section{Yi Ba}

Tianjin Cancer Hospital

\section{Xin-bo Wang}

Eastern Theater General Hospital of Chinese PLA

\section{YeXu}

Shanghai Cancer Hospital: Fudan University Shanghai Cancer Center

\section{Lin Zhou}

302 Military Hospital of China: 5th Medical Center of Chinese PLA General Hospital

\section{Ke-feng Ding}

The Second Affiliated Hospital of Zhejiang University

\section{Yan-ru Qin}

The First Affiliated Hospital of Zhejiang University

\section{Shu-jun Yang}

Henan Cancer Hospital

\section{Yan-ping Zhu}

Nanjing Personal Oncology Biological Technology Co. Ltd 


\section{Research Article}

Keywords: clinical study, patient-derived tumor xenograft, advanced gastrointestinal carcinomas, personalized medication

Posted Date: February 19th, 2021

DOl: https://doi.org/10.21203/rs.3.rs-212867/v1

License: (c) (i) This work is licensed under a Creative Commons Attribution 4.0 International License. Read Full License

Version of Record: A version of this preprint was published at Journal of Cancer Research and Clinical Oncology on April 17th, 2021. See the published version at https://doi.org/10.1007/s00432-021-03639-x. 


\section{Abstract}

Background: Establish patient-derived tumor xenograft (PDTX) from advanced GICs and assess the clinical value and applicability of PDTX for the treatment of advanced GICs.

Method: Patients with advanced GICs were enrolled in a registered multi-center clinical study (ChiCTROOC-17012731). The performance of PDTX were evaluated includes: analyzing factors that affect the engraftment rate, comparing the histological consistency between primary tumors and tumorgrafts, examining the concordance between the drug effectiveness in PDTXs and clinical responses, and Identifying genetic variants and other factors associated with prognosis.

Results: Thirty-three patients were enrolled in the study with the engraftment rate of $75.8 \%(25 / 33)$. The successfulness of engraftment was independent of age, cancer types, pathological stages of tumors, and particularly sampling methods. Tumorgrafts kept same histopathological characteristics as primary tumors. Forty-nine regimens involving twenty-eight drugs were tested in seventeen tumorgrafts. The median time for drug testing was 134.5 day. The follow up information of 10 regimens from 9 patients were obtained. The concordance of drug effectiveness in PDTXs and clinical responses was $100 \%$. The tumor mutation burden (TMB) was correlated with the effectiveness of single drug regimens, while the outgrowth time of tumorgrafts was associated with the effectiveness of combined regimens.

Conclusion: The engraftment rate in advanced GICs is higher than other cancers and meets the general acceptable standard of applying personalized therapeutic strategies. Tumorgrafts from PDTX kept attributes of primary tumor. Predictions from PDTX modeling highly agree with the clinical drug responses. PDTX may already be clinical applicable for the personalized medication in advanced GICs.

\section{Full Text}

Due to technical limitations, full-text HTML conversion of this manuscript could not be completed. However, the manuscript can be downloaded and accessed as a PDF.

\section{Figures}




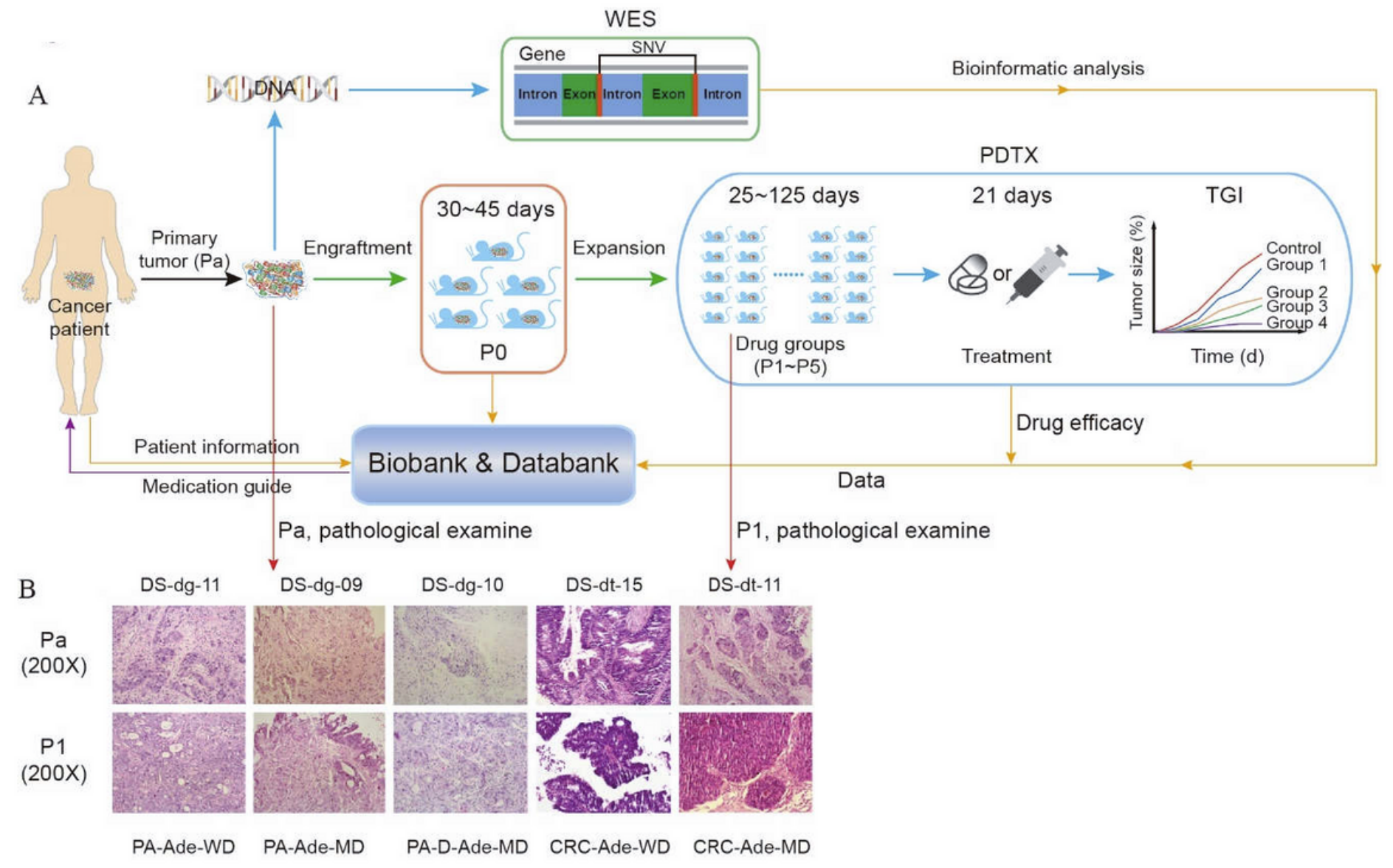

Figure 1

the establishment of PDTX in advanced gastrointestinal cancers. A. schematic illustration of PDTX modeling. B. Pathological comparation between the primary tumor $(\mathrm{Pa})$ and the first passage of tumorgafts (P0) from various types of cancers. Abbreviations: WES: Whole Exon Sequencing; SNV: Single Nucleotide Variation; PA: Pancreatic Cancer; Ade: Adenocarcinoma; WD: Well Differentiated; MD: Moderate Differentiated. 
A 品

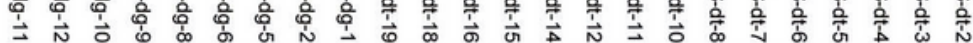
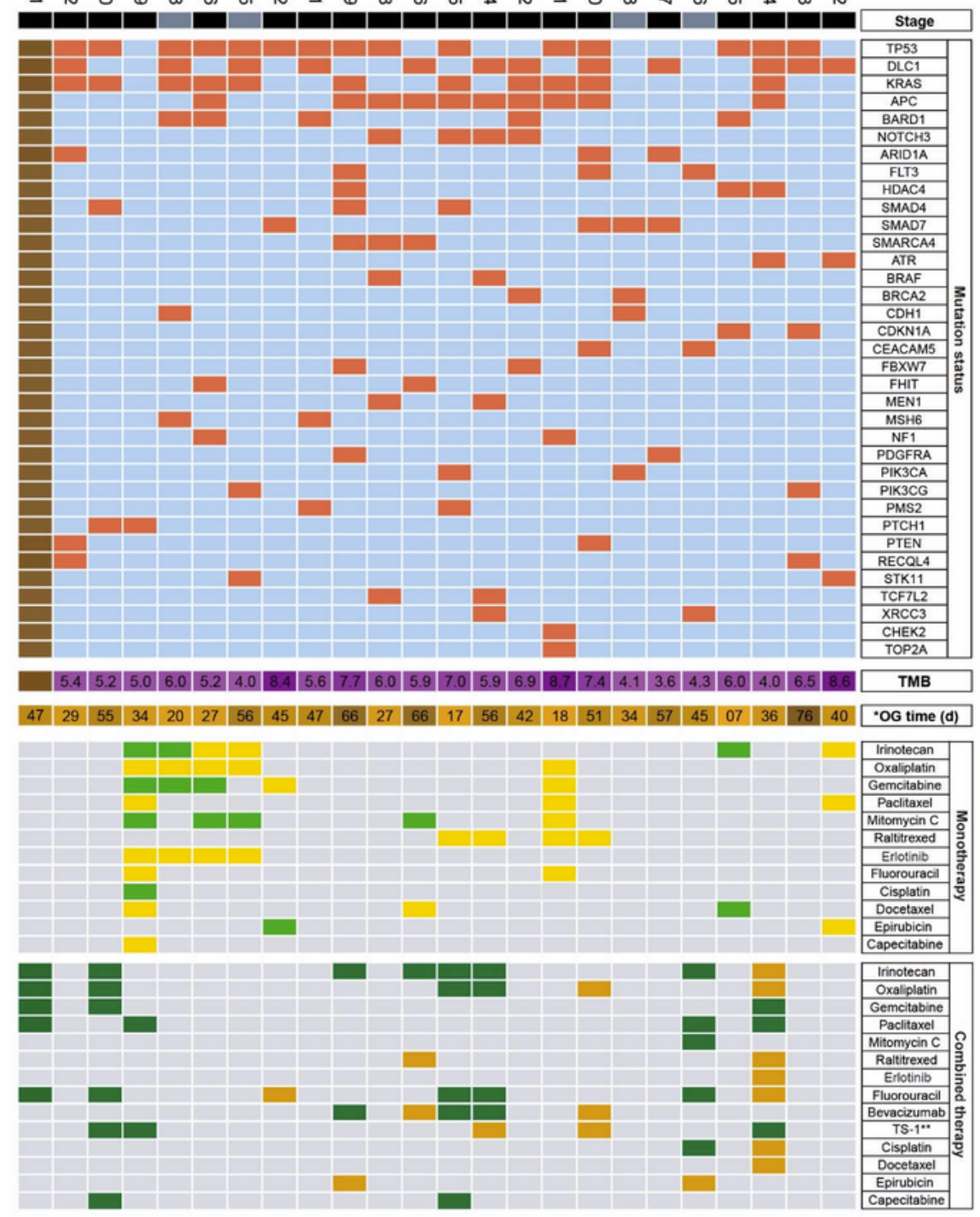

Stage IV $\square$ Mutated $\square T G I \geq 60 \% \square T G I \geq 60 \% \square$ Not tested $\square$ Non-effective $\quad$ OG time $=$ Outgrowth time

B

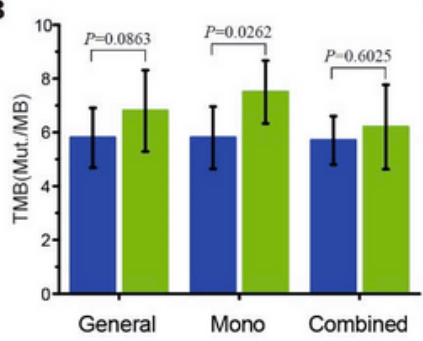

C 80

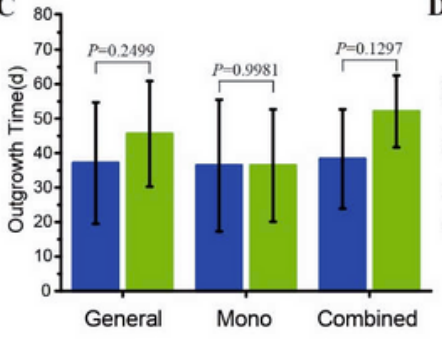

D

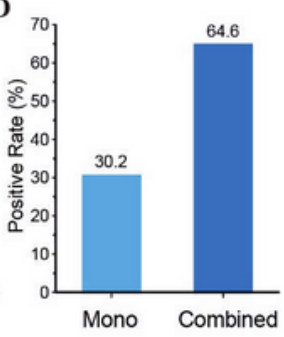

\section{Figure 2}

Mutational landscape of tumorgrafts and drug efficacy. A. Major driver mutations discovered in tumorgrafts and corresponding drug responses. B. Correlation of tumor mutation burden (TMB) with the drug effects. C. Correlation of tumor formation time with the drug effects. D. The comparison of effective rate between single-drug and combined-drug regimens. 
A

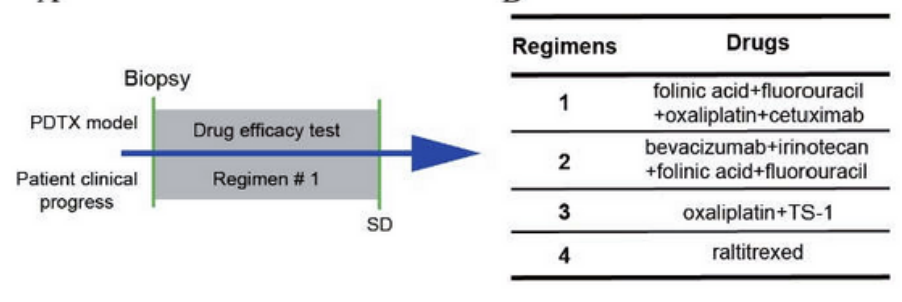

E

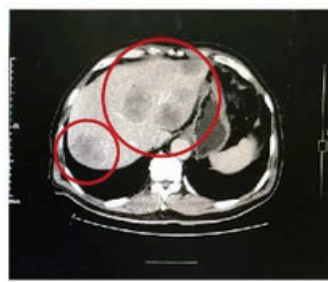

Before treatment

I

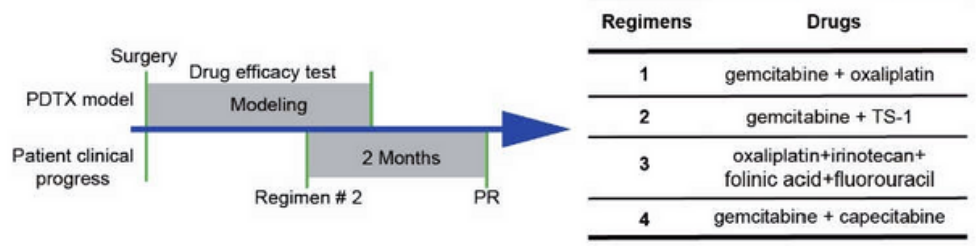

M

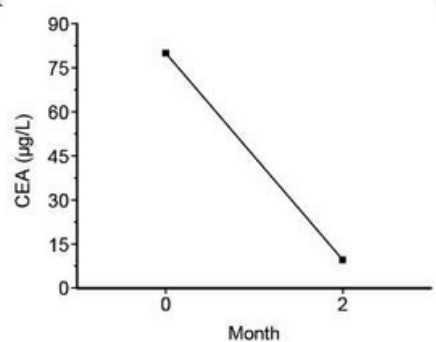

F

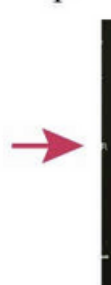

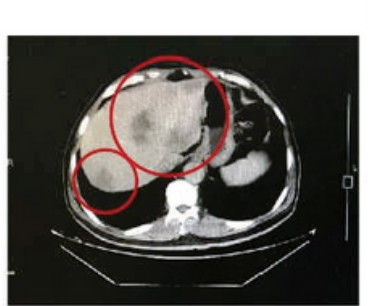

After treatment
C

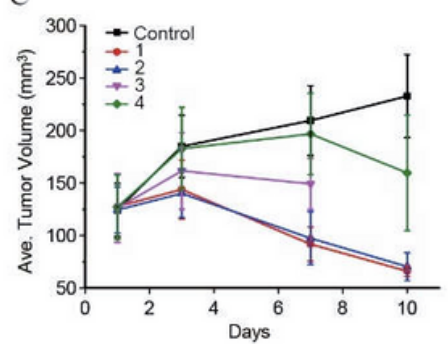

G
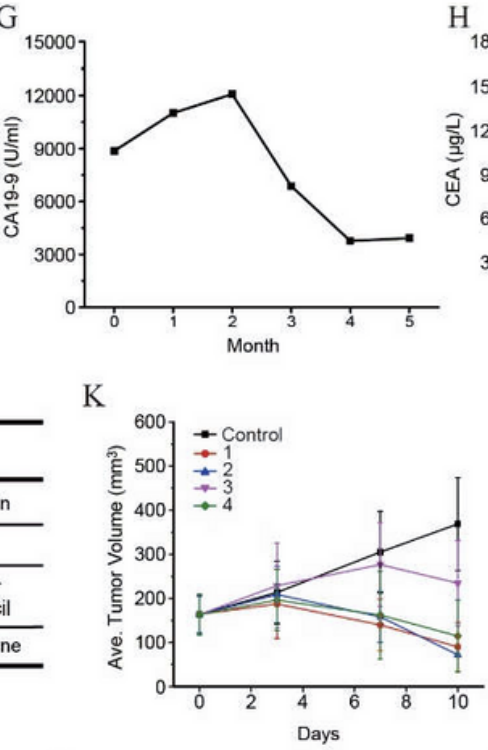

$\mathrm{O}$

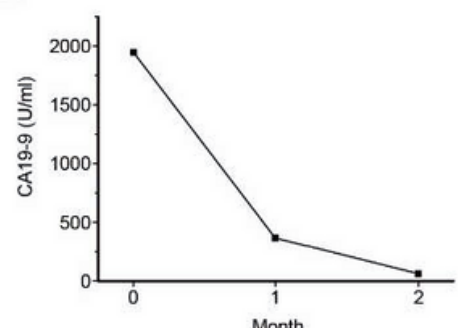

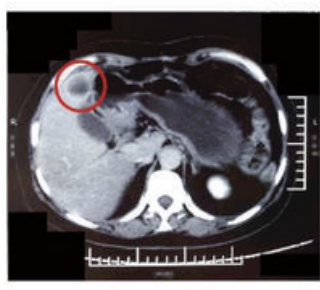

Before treatment

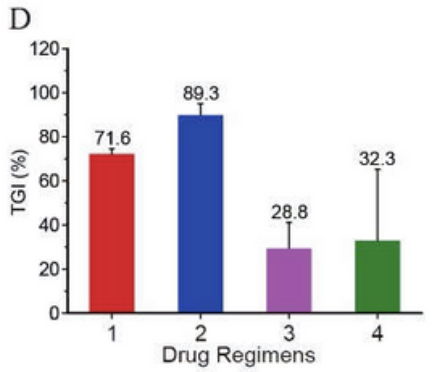

$\mathrm{H}$

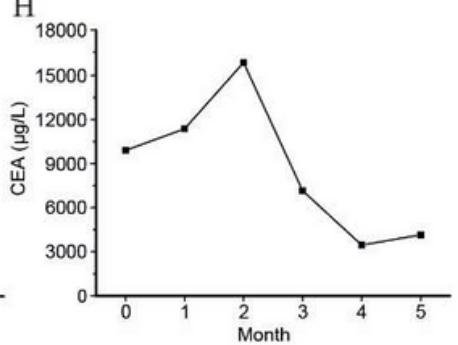

L

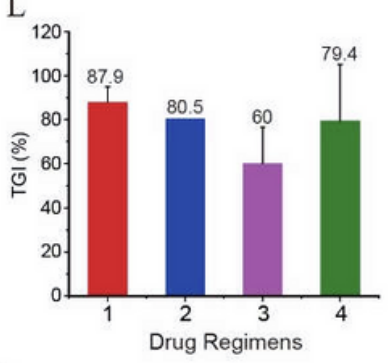

P

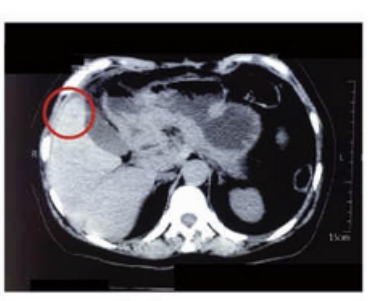

After treatment

\section{Figure 3}

Typical cases of PDTX in advanced GICs. A-H, Advance CRC; I-P, Advance PDAC. A and I, Schematic time frame of modeling in each case. B and J, Drug regimens tested in each case. C, D and K, L, Growth inhibition of tumorgrafts for each drug regimen. $\mathrm{E}, \mathrm{F}$ and $\mathrm{M}, \mathrm{N}$, Biomarker changes before and aftertreatment. G, H and O, P, CT scans of tumors before and aftertreatment. Red circles indicate the locations of tumors. CEA= Carcinoembryonic antigen; CA19-9= carbohydrate antigen 19-9. 